\title{
Pd(II)-Ni(II) pyrazolate framework as active and recyclable catalyst for the hydroamination of terminal alkynes
}

\author{
Francisco G. Cirujano $^{\mathrm{a} *}$, Elena López-Maya ${ }^{\mathrm{b}}$, J. A. R. Navarro ${ }^{\mathrm{b}}$, and Dirk E. De Vos ${ }^{\mathrm{a}}$ \\ ${ }^{a}$ Centre for Surface Chemistry and Catalysis, Department of Microbial and Molecular Systems (M2S), \\ KU Leuven, Celestijnenlaan 200F, 3001 Leuven (Belgium). \\ E-mail: francisco.garcia@kuleuven.be \\ bDepartamento de Química Inorgánica, Universidad de Granada, Av. Fuentenueva S/N, 18071 Granada \\ (Spain)
}

\begin{abstract}
Well-dispersed $\left[\mathrm{Pd}\left(\mathrm{NH}_{3}\right)_{4}\right]^{2+}$ sites have been introduced in the defective nickel pyrazolate porous framework, known as K@NiBDP. The resulting porous crystalline material shows a superior catalytic behavior for $\mathrm{C}-\mathrm{N}$ bond formations than $\left[\mathrm{Pd}\left(\mathrm{NH}_{3}\right)_{4}\right]^{2+}$ containing zeolite $\mathrm{HY}$. The palladium exchanged catalyst, Pd@NiBDP, is able to promote both intra- and intermolecular hydroamination of terminal alkynes with excellent activity, reusability and selectivity towards the corresponding indole or imine vs. the hydration of the alkyne. The same Pd species introduced in the microporous acid HY zeolite favors the hydration of the terminal alkyne towards the corresponding ketone, instead of the addition of the amine to the triple bond.
\end{abstract}

\section{Keywords}

Hydroamination, Heterogeneous catalysis, Metal-Organic Framework

\section{Introduction}

The pharmaceutical and chemical industries are continuously searching for processes with a lower environmental impact, not only by the use of greener solvents and reactants, but also limiting the number of steps while safeguarding the purity and safety of high-quality but affordable therapeutic molecules. In particular, nitrogen containing molecules are pharmaceutically interesting compounds due to their potent physiological activity and new strategies with reduced numbers of transformation steps and purification procedures, lower costs, and minimized chemical waste are still in high demand [1]. For instance, Ackermann et al. have shown that homogeneous transition-metal-catalysed $\mathrm{C}-\mathrm{H}$ activation strategies enabled the generation of the heterocyclic indole moiety directly from protected anilines [2]. Even so, the translation of the latest breakthroughs of such homogeneous catalysed reactions to the industrial pharmaceutical production is unsolved, due to complicate product purification, deactivation and moderate selectivity. Given the solid nature of almost all large-scale industrial catalysts, the convenient use of a heterogeneous catalyst (in the targeted hypothesis of negligible leaching) allows to at least avoid the additional unit operation of adsorptive purification for removal of the metal catalyst [3]. Indeed, purification of the product and removal of the homogeneous catalyst are much simpler or avoided; a simple filtration suffices, thus one does not have to do a charcoal filtration, or even a chromatographic purification with state-of-the-art homogeneous catalysts described. In this sense, the family of microporous solids, i.e. zeolites and metal-organic frameworks, presents an important advancement with respect to traditional state-of-the-art homogeneous catalysts, such us metal salts [4a]. This is mainly due to the immobilization of the catalytic active sites in the porous host material to overcome the metal losses and deactivation occurring with state-of-the-art homogeneous catalysts, since metal residues are totally unallowed in pharmaceuticals, i.e. downstream processing imposes $\leq 20 \mathrm{ppm}$ of Platinum-Group Metals. With the aim of immobilizing homogeneous Pd catalytic species for organic synthesis, many reaction media and solid matrices have been already explored [4b-c]. In this work, zeolites and metal-organic frameworks are compared as porous solid matrices of palladium catalytic species for the synthesis of $N$ containing molecules, i.e. imines and enamines. On the one hand, palladium species on zeolite cages have been reported during the last decades as heterogeneous catalysts for the synthesis of (substituted) indoles 
or other substituted aromatic molecules, due to the porous and stable nature of these crystalline solids [5]. However, the limited metal uptake and diffusion restrictions of large reactants and/or product molecules are often an issue, even for large pore zeolites (*BEA, FAU ...). On the other hand, Metal-Organic Frameworks (MOFs) presents additional advancements with respect to traditional state-of-the-art heterogeneous porous catalysts, such us zeolites or porous carbons. In particular MOFs: avoid neutralization/separation operations due to the simple recovery of the solid catalyst, decreasing leaching by providing strong metal-ligand bonds in contrast to traditional porous carbons and offers a much higher activity than homogeneous catalysts due to ultrastable catalytic site isolation, avoiding self-deactivation and ensuring adequate diffusion through the pores in contrast to zeolites. Surprisingly, the performance of state-of-the-art Pd containing MOFs, either appended to the N-containing linker or directly confined in the pores, has been rarely studied as catalysts in $\mathrm{C}-\mathrm{H}$ activation and $\mathrm{C}-\mathrm{N}$ bond formation reactions [6]. Recently, new porous materials such as the robust nickel pyrazolate frameworks containing linker vacancies have been prepared in our group. In this solid, K@NiBDP (BDP = 1,4-bis(pyrazol-4yl)benzene), the pyrazolate vacancies (missing linker defects) are substituted by exposed hydroxides and extraframework cations, i.e. $\mathrm{K}$ or $\mathrm{Ba}$ [7]. After ion-exchange, these bimetallic MOFs have been demonstrated to be highly porous and stable crystalline solids with applications in adsorption, ionconduction and acid-base catalysis. Therefore the NiBDP is a potential porous platform to host other type of guest species, such as the catalytically active $\left[\mathrm{Pd}\left(\mathrm{NH}_{3}\right)_{4}\right]^{2+}$, already incorporated in zeolites [8]. Encouraged by recent reports of Pd MOF catalysts obtained by ion-exchange [9], and with the aim of improving the stability of the resultant heterogeneous catalyst, as occurs in zeolites, here we incorporate the $\mathrm{Pd}\left(\mathrm{NH}_{3}\right)_{4} \mathrm{Cl}_{2}$ palladium salt through ion exchange into a highly robust Ni pyrazolate framework MOF. The resulting heterogeneous catalyst is targeted to be used in hydroamination reactions under mild and non-inert or anhydrous conditions. This type of organic transformation is interesting by itself, due to the fact that terminal alkynes are versatile intermediates that allow the access to $\mathrm{N}$ containing molecules, such as imines or amines, through the addition of amine nucleophiles in the presence of a catalyst [10]. In this work we employ a novel Pd-Ni pyrazolate framework as heterogeneous catalyst for this type of C-H activation of terminal alkynes in the presence of primary amines. Moreover, a comparison between inorganic (HY) and metal-organic (NiBDP) microporous solids as matrix for the confined palladium active sites $\left[\mathrm{Pd}\left(\mathrm{NH}_{3}\right)_{4}\right]^{2+}$ will be established both for the inter- and intra-molecular hydroamination reactions of terminal alkynes with anilines, as summarized in the Scheme 1.

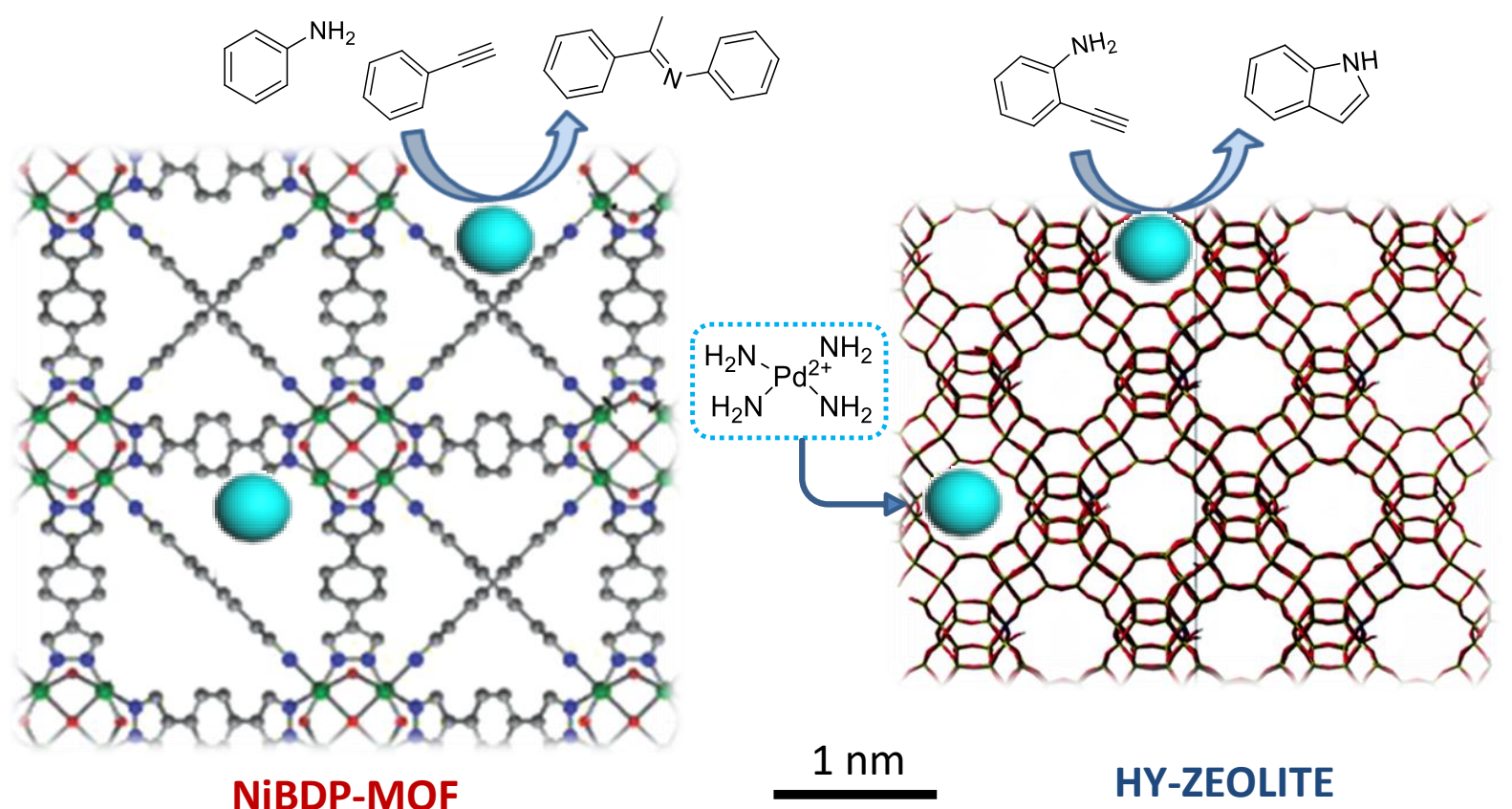

Scheme 1. Intermolecular (left) and intramolecular (right) hydroamination between anilines and phenylacetylene derivatives using catalytic amounts of $\left[\mathrm{Pd}\left(\mathrm{NH}_{3}\right)_{4}\right]^{2+}$ in the porous NiBDP MOF or HY zeolite. 


\section{Experimental Results}

\subsection{Catalyst synthesis}

In order to obtain the highly porous and defective K@NiBDP metal-organic framework that is employed here as a support of the palladium sites, a KOH treatment of the parent NiBDP MOF was performed to generate pyrazolate vacancies and extraframework $\mathrm{K}^{+}$cations, according to reported procedures [7]. Briefly, $0.055 \mathrm{mmol}$ of NiBDP were suspended in $0.35 \mathrm{M} \mathrm{KOH}$ absolute ethanol solution $(5.5 \mathrm{~mL})$ and stirred overnight at room temperature. The $\mathrm{Cu} @ \mathrm{NiBDP}$ was prepared for comparison purposes by suspending $100 \mathrm{mg}$ of NiBDP@K in $12 \mathrm{~mL}$ of $0.1 \mathrm{M}$ methanolic solution of $\mathrm{Cu}\left(\mathrm{ClO}_{4}\right)_{2}$ and stirring for $16 \mathrm{~h}$ at room temperature. The desired Pd containing MOFs or zeolites were prepared adapting the procedures already reported $[8 \mathrm{~b}, 9 \mathrm{~b}, 10 \mathrm{c}]$. As illustrated in the Scheme 2, a solution of $20 \mathrm{mg}(0.08 \mathrm{mmol})$ of $\mathrm{Pd}\left(\mathrm{NH}_{3}\right)_{4} \mathrm{Cl}_{2}$ in $2 \mathrm{ml}$ of distilled water, was added to $100 \mathrm{mg}$ of NiBDP@K solid under stirring conditions $(250 \mathrm{rpm})$ at room temperature overnight. The $\left[\mathrm{Pd}\left(\mathrm{NH}_{3}\right)_{4}\right]^{2+} @ \mathrm{NiBDP}$ solid (here denoted briefly as Pd@NiBDP) was isolated by centrifugation and washed with water two times and once with diethyl ether. Finally, the solid was dried with a flow of nitrogen at room temperature and used as catalyst in the hydroamination reaction.

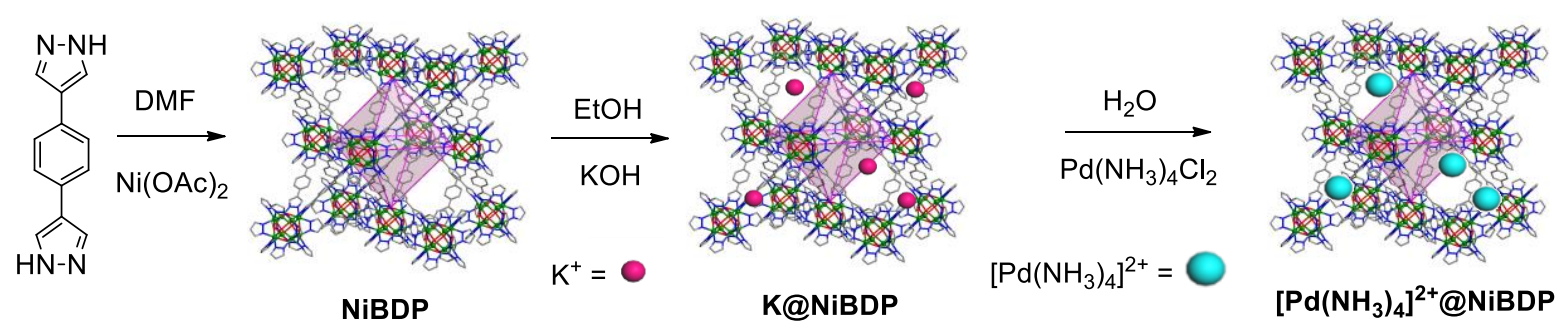

Scheme 2. $\left[\mathrm{Pd}\left(\mathrm{NH}_{3}\right)_{4}\right]^{2+}$ containing NiBDP catalyst prepared by ion exchange of K@ NiBDP.

Different characterization techniques have been employed in order to address the composition, structure and stability of the new MOF heterogeneous catalyst prepared. The thermogravimetric analyses (TGA) results of Figure S1 show that both the starting defective MOF (K@NiBDP) and the palladium exchanged one (Pd@NiBDP) are thermally stable up to $300{ }^{\circ} \mathrm{C}$, starting to decompose above such temperatures, as indicated by the weight loss corresponding to the burning of the pyrazolate linker. The presence of $\left.\mathrm{Pd}\left(\mathrm{NH}_{3}\right)_{4}\right) \mathrm{Cl}_{2}$ in the pores of Pd@NiBDP is also deduced from the higher amount of inorganic residue observed in the thermo-gravimetric analysis, which corresponds to $\mathrm{PdO}$ in addition to the expected $\mathrm{NiO}$. From the organic and inorganic weight losses observed in Figure S1, the calculated inorganic oxides coming from Pd@NiBDP are 37.4\%, slightly higher than the one expected: $(\mathrm{NiO})_{8}(\mathrm{PdO})_{0.5}(36.9 \%)$. Elemental analysis by inductivelly coupled plasma-mass spectrometry (ICP-MS) of the Pd@NiBDP

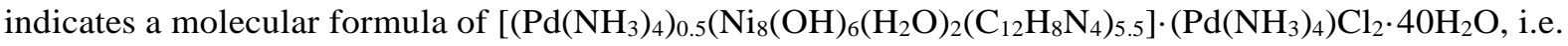
5.6 wt.\% $\mathrm{Pd}$, in agreement with the $\mathrm{Ni}: \mathrm{Pd}$ ratio of 8:1.5 and only traces of $\mathrm{K}$. The infrared spectra of Figure S2 further suggest the presence of the $\mathrm{Pd}\left(\mathrm{NH}_{3}\right)_{4}{ }^{2+}$ in the porous NiBDP matrix. In particular, the $\mathrm{NH}_{3}$ antisymmetric deformation around $1600 \mathrm{~cm}^{-1}$ informs about the presence of the $\mathrm{Pd}\left(\mathrm{NH}_{3}\right)_{4} \mathrm{Cl}_{2}$ in the NiBDP solid [11]. Finally, the similar (green) color and UV/Vis absorption spectra (see Figure S3) of both K@NiBDP and Pd@NiBDP, does not suggest the initial presence of PdO and/or Pd nanoparticles in the as-prepared Pd@NiBDP catalyst, although it is difficult to obtain further information due to the MOF absorption in that region of the spectra.

\subsection{Catalytic performance in hydroamination of alkynes}

The resulting Pd@NiBDP solid was employed as heterogeneous catalyst for the hydroamination reactions proposed in Scheme 1. We choose the model compound 2-ethynylaniline in order to study the reactivity of this catalyst in the intramolecular hydroamination of the terminal alkyne with the primary amine in ortho- position. The catalytic tests were carried out by dissolving $1 \mathrm{mmol}$ of the reagents in $3 \mathrm{ml}$ of toluene in the presence of 50 $\mathrm{mg}(0.02 \mathrm{mmol} \mathrm{Pd})$ of Pd@NiBDP and heating it at $80{ }^{\circ} \mathrm{C}$ under stirring conditions. After $48 \mathrm{~h}$, the highest conversion of 2-ethynylaniline $(80 \%)$ was obtained in the presence of the Pd@NiBDP MOF, with a $64 \%$ 
selectivity towards the indole hydroamination product $\mathbf{1}$ (see Figure 1a). We also wanted to compare the catalytic performance of this Pd@NiBDP catalyst with well-established Pd@HY zeolite prepared under the same conditions (see part 2.1 of the experimental results). The catalytic activity of both Pd containing porous solids is superior to the homogeneous $\left[\mathrm{Pd}\left(\mathrm{NH}_{3}\right)_{4}\right] \mathrm{Cl}_{2}$ salt, probably due to its poor solubility in toluene [10c], and much higher than the parent K@NiBDP or Cu@NiBDP, which produce less than 20\% conversion. A better catalytic performance of the Pd@NiBDP with respect to the Pd@HY was obtained in the case of the intermolecular hydroamination of phenylacetylene with aniline, where the selectivity to the imine 4 was $68 \%$ with respect to the only $13 \%$ obtained in the case of the HY zeolite, at complete conversion of phenylacetylene (see Figure 1b). This may be related to the better accessibility of the reagents to the inner Pd sites and the preclude formation of acetophenone (from the hydration of the alkyne), in the case of the MOF catalyst. While no hydration of phenylacetylene is observed with the NiBDP, in the case of the HY zeolite, acetophenone was obtained in $24 \%$ yield even in the absence of palladium, when equimolar amounts of water and phenylacetylene are heated at 80 ${ }^{\circ} \mathrm{C}$ in toluene. This should be attributed to the presence of active acid sites in the zeolite [12a], as has been previously reported for homogeneous acid species [12b-d].
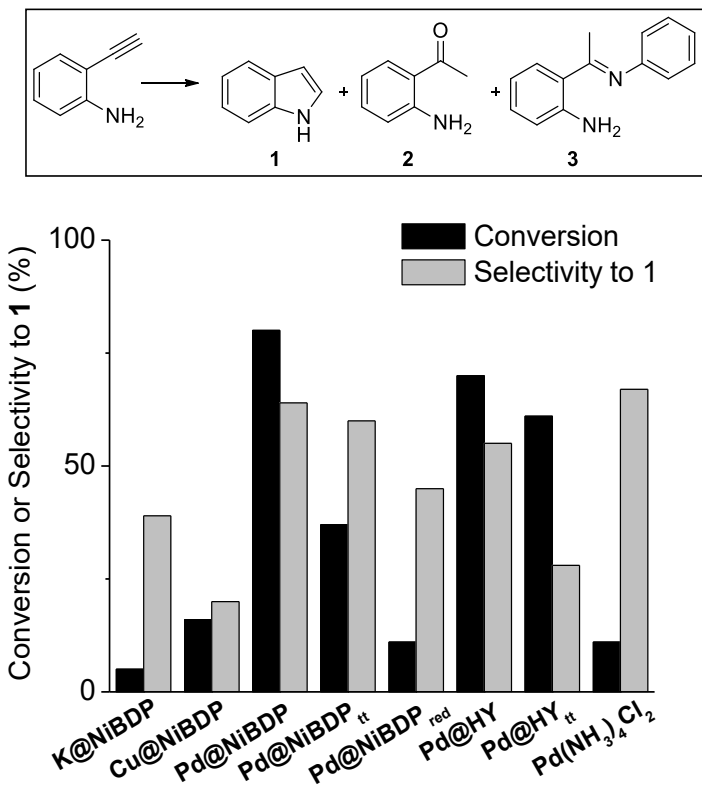

(a)
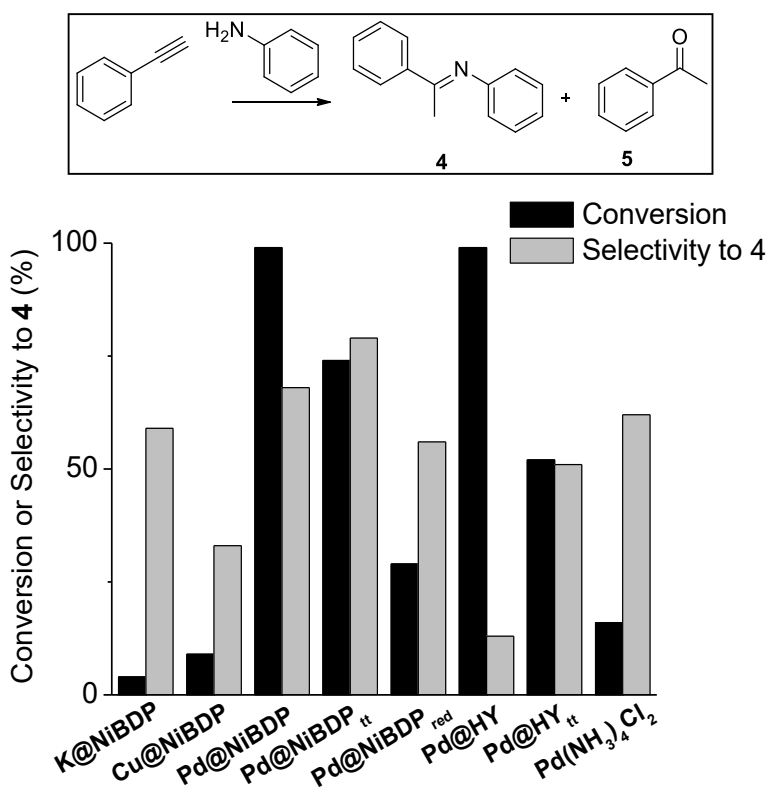

(b)

Figure 1. Intramolecular (a) and intermolecular (b) hydroamination of terminal alkynes with anilines using $\mathrm{Pd}\left(\mathrm{NH}_{3}\right)_{2} \mathrm{Cl}_{2}$ exchanged porous solids. Reaction conditions: $1 \mathrm{mmol}$ of reactant(s) and $50 \mathrm{mg}$ of catalyst in $3 \mathrm{ml}$ of toluene at $80^{\circ} \mathrm{C}$ under stirring for $48 \mathrm{~h}$.

The $\left[\mathrm{Pd}\left(\mathrm{NH}_{3}\right)_{4}\right]^{2+}$ seems to be the catalytic active sites in the hydroamination of the terminal alkynes, since the reaction with the amine proceed after contacting the reagent(s) with the Pd@NiBDP or Pd@HY solids and no induction periods were observed for intra- or intermolecular hydroamination (see Figure $2 \mathrm{a}$ and $\mathrm{b}$ ). A thermal treatment at $200{ }^{\circ} \mathrm{C}$ overnight (see $\mathrm{Pd} @ \mathrm{NiBDP}_{\mathrm{tt}}$ and $\mathrm{Pd} @ \mathrm{HY}_{\mathrm{tt}}$ in Figure 1) or a reduction with hydrogen $\left(\mathrm{Pd} @ \mathrm{NiBDP}_{\text {red }}\right.$ and $\mathrm{Pd} @ \mathrm{HY}_{\text {red }}$ in Figure 1) reduces the activity of the as prepared materials in both intra and intermolecular hydroamination. The brownish or black color of the solid after the thermal (tt) or reduction (red) treatment indicates the partial agglomeration and reduction of the palladium (II) species after the removal of $\mathrm{NH}_{3}$ ligands. This suggest the higher activity of cationic $\operatorname{Pd}(\mathrm{II})$ species with respect to $\operatorname{Pd}(0)$ nanoparticles in this reactions. In general, catalytic amounts of $\left[\mathrm{Pd}\left(\mathrm{NH}_{3}\right)_{4}\right]^{2+}$ incorporated in the K@NiBDP MOF (see Pd@ NiBDP in Figure $2 \mathrm{c}$ and $\mathrm{d}$ ), shows higher selectivity towards the hydroamination product $\mathbf{1}$ or $\mathbf{4}$ than when it is in zeolite HY (Pd@HY in Figure 2c and d). This is due to the fact that the acid HY zeolites tested (comprising Si/Al ratios of 2.5, 15, 30 and 40) favors the hydration of the terminal alkyne, obtaining a higher amount of 2aminoacetophenone (2) or acetophenone (5) vs. the hydroamination product (1 or $\mathbf{4})$. Thus, the conversion of phenylacetylene is higher for the zeolites (see CBV samples in in Figure 2b), but the selectivity towards the imine 4 is much lower than with the Pd@NiBDP (see Figure 2d), due to the favored hydration reaction of phenylacetylene when using the hydrophilic zeolites. Moreover, for the MOF catalyst the selectivity to hydroamination products $\mathbf{1}$ and $\mathbf{4}$ increases upon increasing the conversion, while the selectivity to $\mathbf{5}$ decreases in 
an almost linear trend in the three cases. One hypothesis for such particular trend is that the acetophenone $\mathbf{5}$ that may be formed at the beginning of the reaction reacts with the aniline present to form the desired imine 4 . In fact, the reaction between 4-methoxyacetophenone and aniline (under the same conditions described for the hydroamination) is 100 times faster in the presence of the Pd@NiBDP with respect to the blank reaction $\left(10^{-2}\right.$ $\mathrm{mmol} / \mathrm{h}$ vs. $\left.10^{-4} \mathrm{mmol} / \mathrm{h}\right)$.
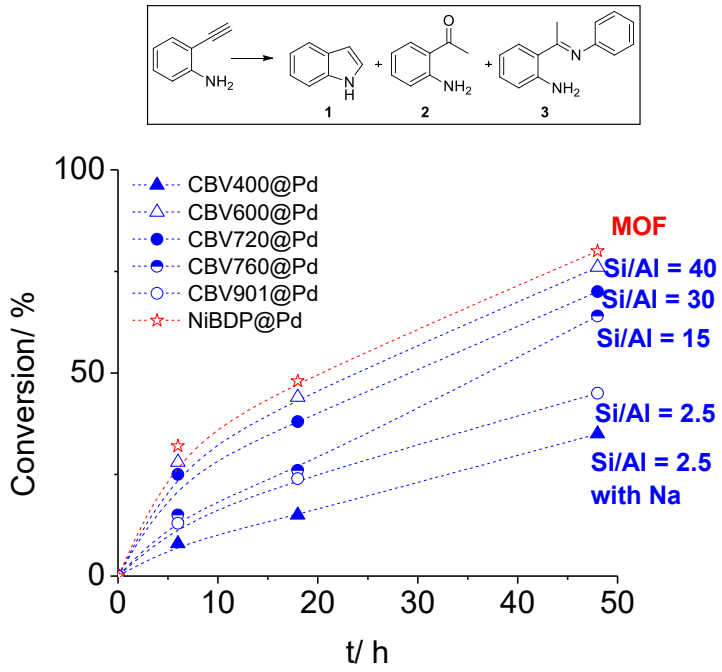

(a)

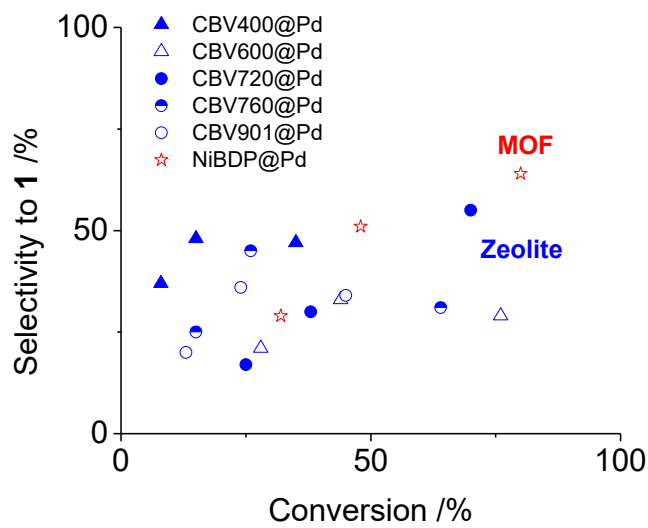

(c)

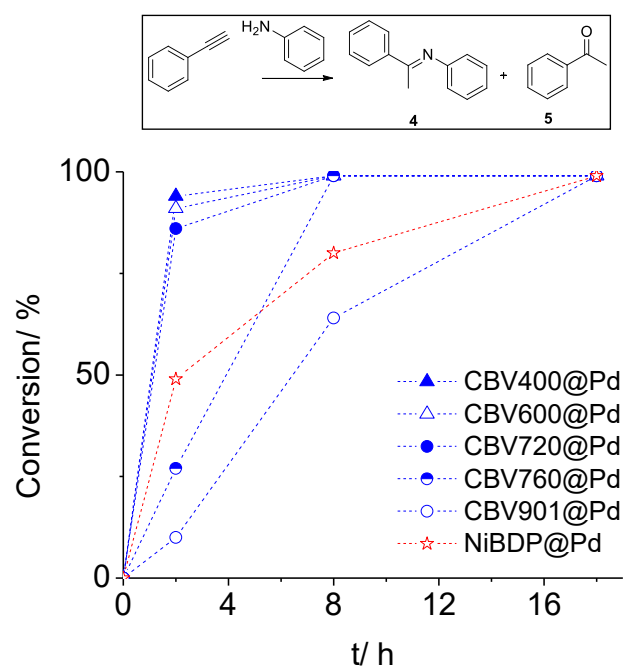

(b)

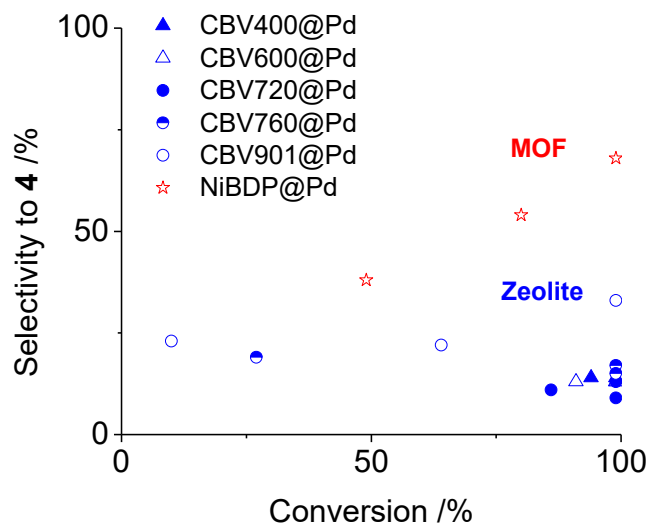

(d)

Figure 2. Conversion-time (a, b) and selectivity-conversion (c, d) to the hydroamination product $\mathbf{1}$ or $\mathbf{4}$ using 50 $\mathrm{mg}$ of catalysts in $3 \mathrm{ml}$ of toluene at $80^{\circ} \mathrm{C}$.

All of the commercial HY zeolites with $\mathrm{Pd}\left(\mathrm{NH}_{3}\right)_{4}{ }^{2+}$ tested promote the hydration of the terminal alkyne with respect to its hydroamination. For the zeolite samples that contains a higher amount of Brønsted acid sites (low Si/Al ratio), the hydration of phenylacetylene is faster than for the more hydrophobic samples (high Si/Al), being acetophenone the main reaction product when using the Pd@HY zeolite (see Figure 3 ). Another reason for the higher hydroamination activity of the Pd containing MOF with respect to the zeolite may be related to the highly porous structure of NiBDP MOF, which contains large pore apertures $(\sim 1.6 \mathrm{~nm})$ with respect to the zeolite structure $(\sim 0.7 \mathrm{~nm})$, allowing for a better diffusion of the bulky products such as compound 4 (see Scheme 1). Although for the intramolecular hydroamination (taking place in the small molecule 2-ethynylaniline to produce compound 1) there are no diffusion problems in the MOF or the zeolite, it is possible to encounter diffusion limitations for the intermolecular hydroamination (between phenylacetylene and aniline) that generates product $\mathbf{4}$ in very limited amounts when using the zeolite Pd@HY but not with the Pd@NiBDP MOF. Apart from this steric effect, the consumption of phenylacetylene into acetophenone can be minimized by treating the Pd@HY zeolite at $200{ }^{\circ} \mathrm{C}$ overnight. This treatment generates a material $\left(\mathrm{Pd} @ \mathrm{HY}_{\mathrm{tt}}\right)$ with decreased water content with 
respect to the as-prepared Pd@HY zeolite (Figure 1). This fact prevents the complete alkyne hydration, increasing the imine 4 selectivity (from $13 \%$ to $51 \%$, at $52 \%$ conversion), which still lower than that obtained with the thermally treated MOF Pd@ NiBDP ${ }_{\mathrm{tt}}(79 \%$ at $74 \%$ conversion).

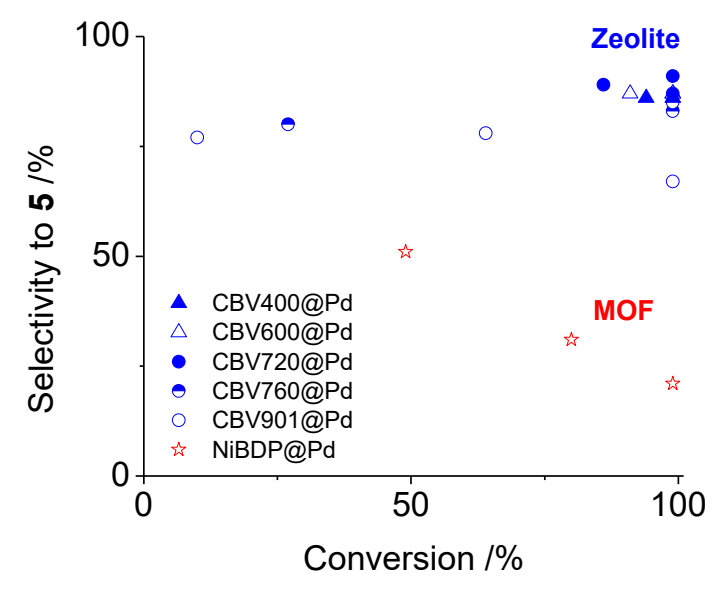

(a)

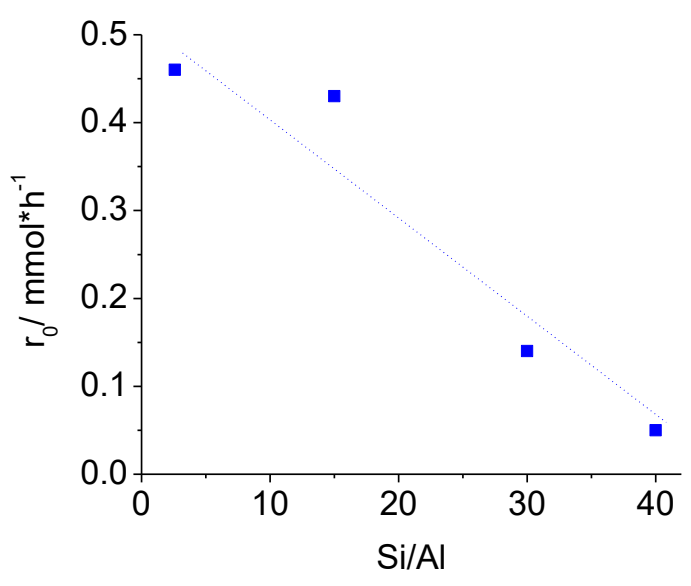

(b)

Figure 3. (a) Selectivity to the hydration product 5 (acetophenone) vs. conversion of phenylacetylene and (b) initial rate for the phenylacetylene conversion in the presence of different HY commercial faujasite zeolites containing $\mathrm{Pd}\left(\mathrm{NH}_{3}\right)_{4}{ }^{2+}$ and a Si/Al ratio of 2.5 (CBV600), 15 (CBV720), 30 (CBV760) and 40 (CBV901).

\subsection{Stability of the MOF catalyst under the reaction conditions}

Different characterization techniques have been employed to confirm the stability of the Pd@NiBDP heterogeneous catalyst. In the first place, the NiBDP crystalline structure withstands the aqueous exchange with $\mathrm{Pd}\left(\mathrm{NH}_{3}\right)_{4} \mathrm{Cl}_{2}$ to a high extent, as can be seen in the Powder X-ray diffraction (XRD) pattern of Figure 4 . The diffraction peaks of the parent K@NiBDP are present in the Pd@NiBDP and no additional phases, such as aggregated palladium species, are observed in agreement with the color of the sample (Figure S3). The Pd@NiBDP structure withstand the reaction conditions (intramolecular hydroamination of 2-ethynylaniline) showing all the characteristic peaks of the fresh sample (compare the violet and magenta signals in Figure 4) and the absence of large palladium particles. However, it is fair to say that a gradual deterioration of the crystalline structure is observed along the synthesis process. In particular, the insertion of Pd in the MOF seems to decrease the particle size due to the broadening of the diffraction peaks, which is confirmed by electronic microscopy (see Figure S5). 


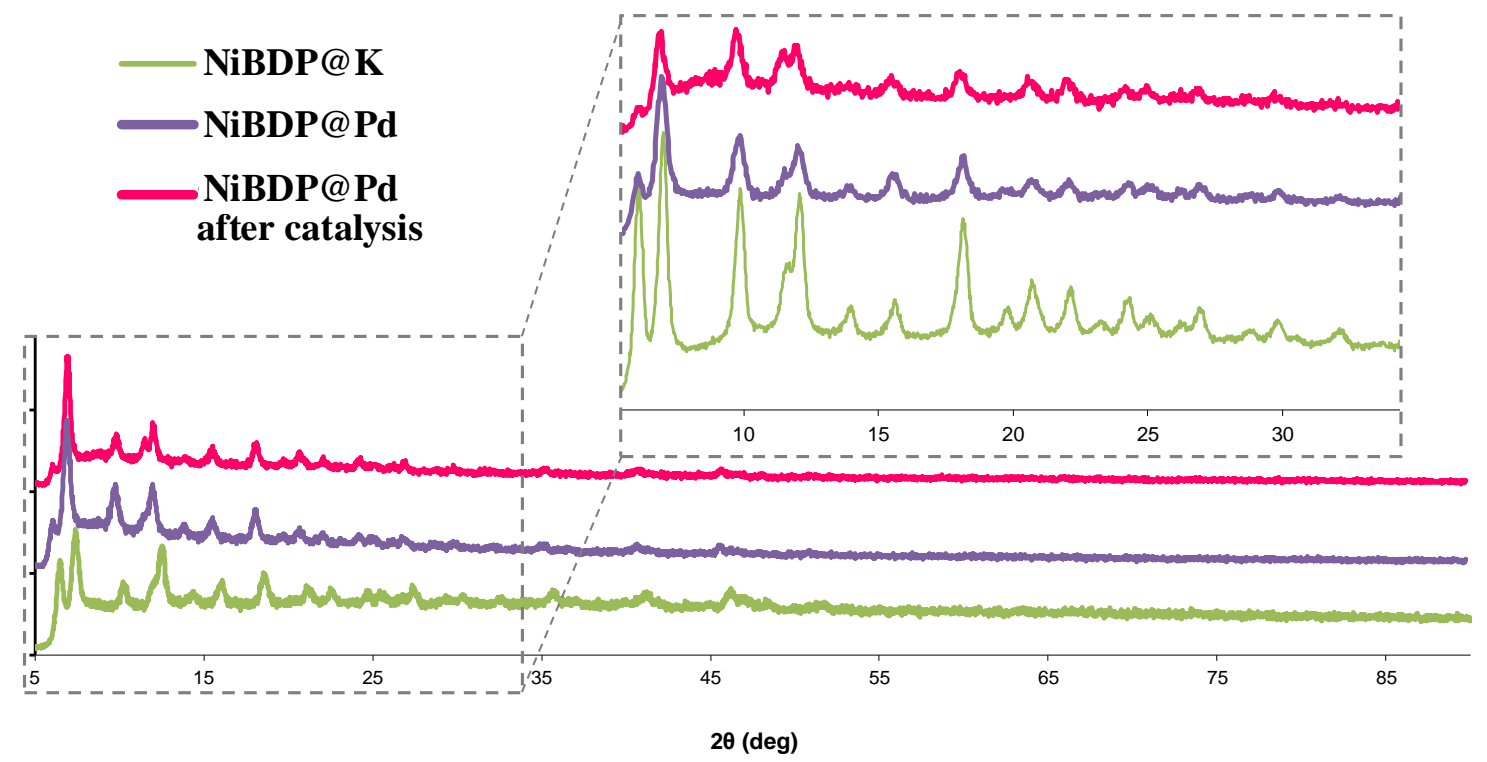

Figure 4. PXRD of samples K@NiBDP (green), Pd@NiBDP “as-prepared” (violet) and Pd@NiBDP after the catalytic test (magenta).

In the second place, X-ray photoelectron spectroscopy (XPS) analysis indicates that the Pd species in the MOF remains in the same oxidation state as in the $\mathrm{Pd}\left(\mathrm{NH}_{3}\right)_{4} \mathrm{Cl}_{2}$ precursor salt (see Figure S4). Moreover, the $\mathrm{N}_{2}$ adsorption isotherms of Figure 5 show that the surface area decreases from $2060 \mathrm{~m}^{2} \mathrm{~g}^{-1}$ for K@ NiBDP to 1131 $\mathrm{m}^{2} \mathrm{~g}^{-1}$ for Pd@NiBDP, probably due to the presence of the occluded palladium species. Inspection of Figure 5 reveals that the $\mathrm{K} @ \mathrm{NiBDP}$ is mesoporous, whereas after $\mathrm{Pd}$ loading, the material is pretty much strictly microporous. This indicates the filling of the micro- and meso- pores in K@NiBDP with more bulky $\mathrm{Pd}\left(\mathrm{NH}_{3}\right)_{4}{ }^{+}$ species during the K exchange process. After the hydroamination reaction the Pd@NiBDP material maintains its porosity with only a slight decrease in its surface area $\left(946 \mathrm{~m}^{2} \mathrm{~g}^{-1}\right)$. Some formation of $\mathrm{Pd}$ aggregates inside the pores of the NiBDP structure may explain the decrease in porosity and would agree with the partial decrease in catalytic activity of this material upon reuses. However, high resolution transmission electron microscopy (HRTEM) imaging and energy-dispersive X-ray spectroscopy (EDX) mapping for $\mathrm{Cl}$, Pd and Ni elements indicates an homogeneous dispersion of Pd in the MOF crystals (Figure S5), before and after the catalytic reaction, indicating the high stability of the Pd active sites in the NiBDP. During the reaction, changes in morphology/aggregation of the particles (see Figures 4 and S5) and its porous texture may account for the extra porosity associated to the hysteresis loop at in the Pd@NiBDP after the reaction. [13]

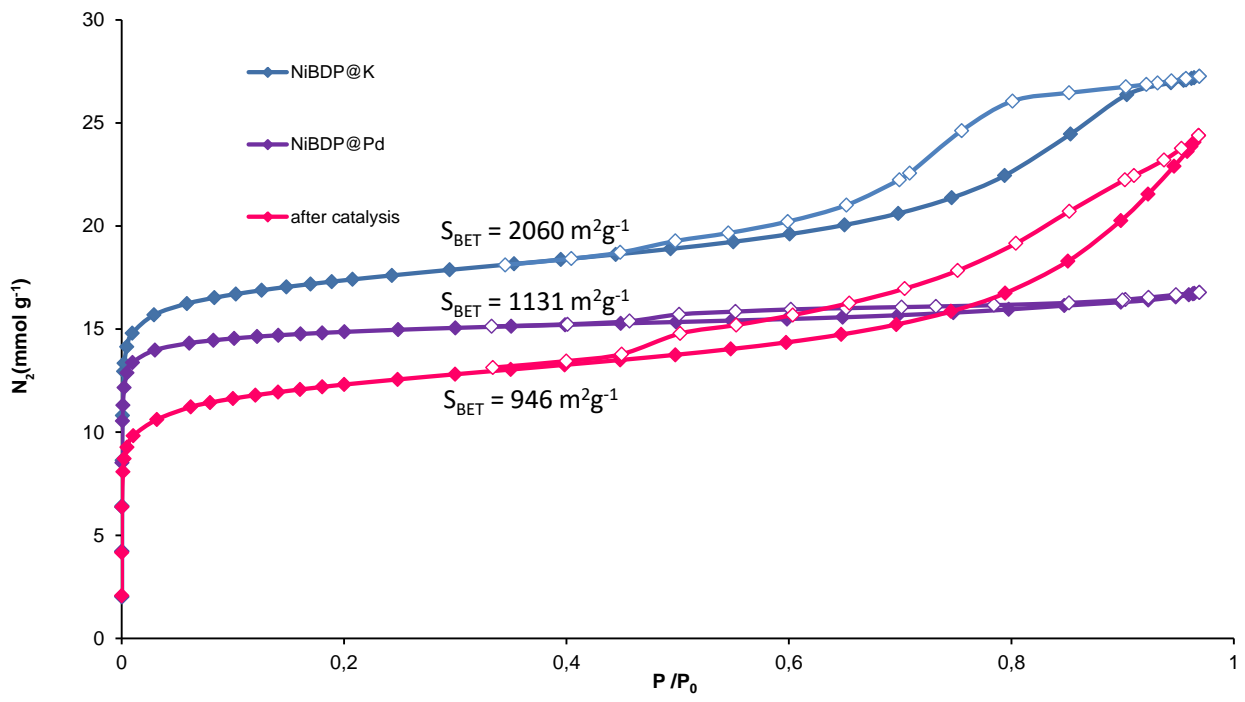

Figure 5. Adsorption isotherms for K@NiBDP, Pd@NiBDP before the reaction and Pd@NiBDP after 2 reuses in the hydroamination of 2-ethynylaniline. 
Despite of the high dispersion and isolation of the Pd catalytic active sites, its stability is of paramount importance in order to minimize leaching and/or deactivation of the active metal $\left(\mathrm{Pd}^{2+}\right)$, especially for the synthesis of pharmaceuticals. Thus, different reuses of the catalyst were performed for the intramolecular hydroamination reaction (see Figure 6a). Although the activity of both MOF and zeolite clearly decreases upon reuses, the reusability of the Pd containing MOF is slightly better than the same species present in the zeolite framework. It seems that the interactions of the cationic Pd species with the $N$-containing pyrazolate and exposed hydroxides of the defective NiBDP framework may be stronger than those with the oxygen of the silicoaluminate framework, due to the preferred soft-soft acid (Pd) - base (N) interactions in the case of the MOF. A hot filtration test after 6 $\mathrm{h}$ of reaction indicates no significant leaching of active species from the solid to the reaction media, since the conversion of the alkyne in the absence of the solid catalyst is very low (see dotted line in Figure 6b). This confirms that the catalytic process is, up to a large extent, heterogeneous. The sum of TONs for each individual recycle of the Pd@NiBDP catalyst results in a cumulative TON of 150, a comparable value to those reported for other Pd systems in literature, such as Pd-NHC. [14]

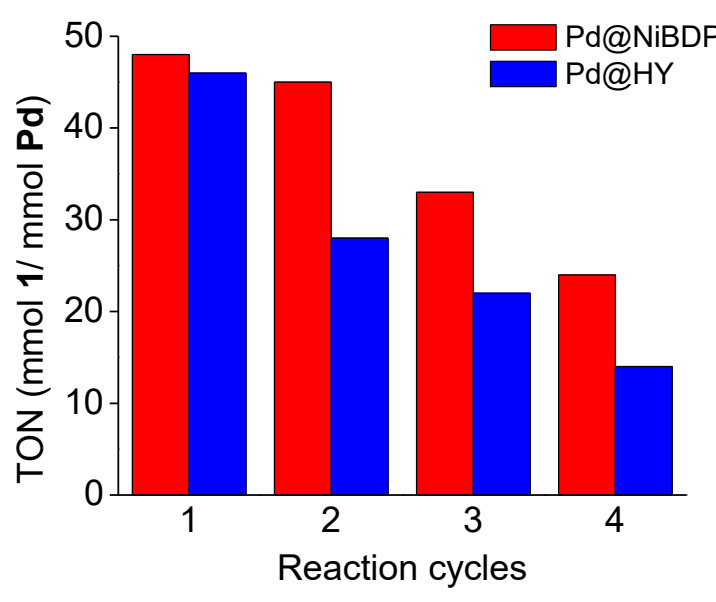

(a)

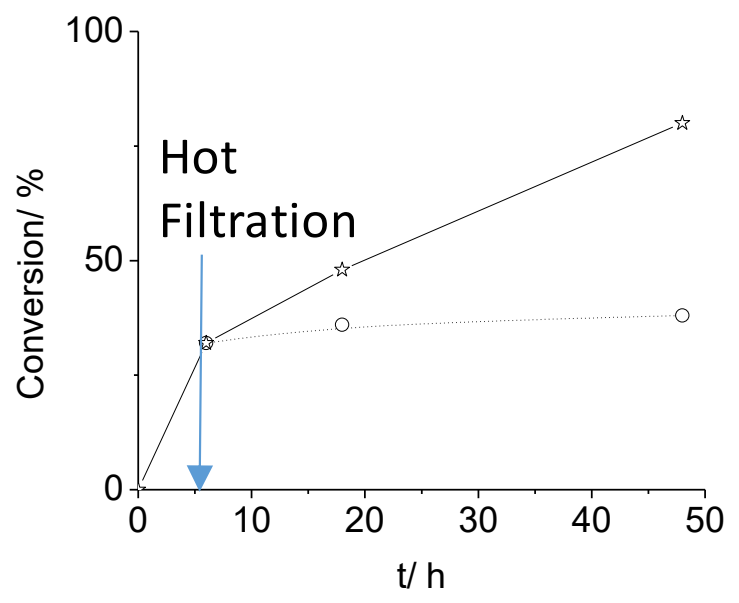

(b)

Figure 6. Reusability of Pd@NiBDP and Pd@HY in 4 reaction cycles (a) and hot-filtration test of Pd@NiBDP (b) in the intramolecular hydroamination of 2-ethenylaniline. Dotted-black lines corresponds to the conversion measured in the filtrates.

A plausible mechanism for the $\mathrm{Pd}^{+2}$ catalysed hydroamination reaction and the hydration (by-product) may proceed through the coordination of palladium sites to the triple bond of the substrate (see Figure 7). Both hydroamination or hydration of the terminal alkyne proceed in the presence of an amine or a water nucleophiles to generate indole or 2-amino acetophenone compounds, respectively. 


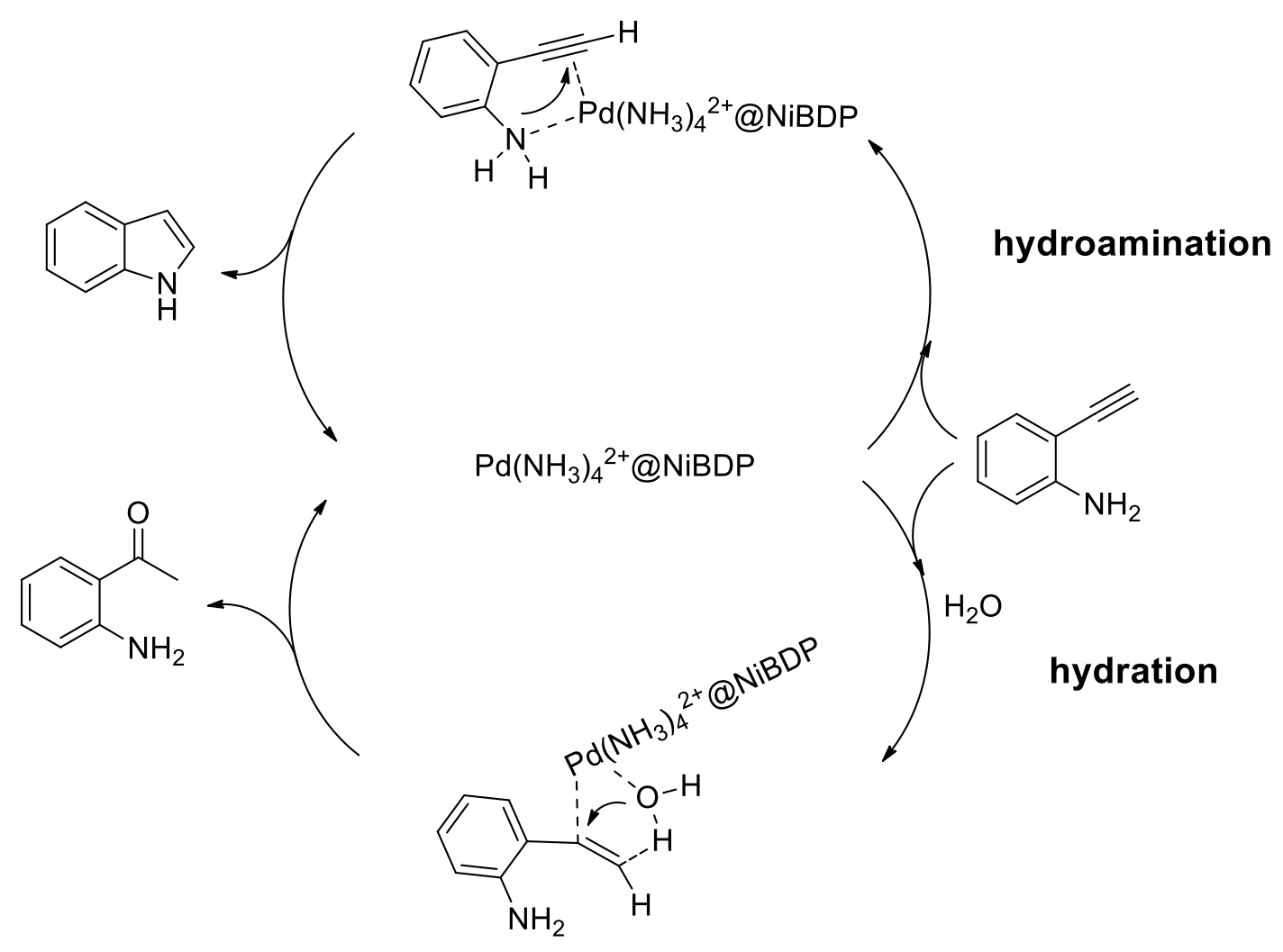

Figure 7. Proposed reaction mechanism for the intramolecular hydroamination or hydration reaction over the Pd@NiBDP.

\section{Conclusions}

This work shows how it is possible to introduce and stabilize cationic palladium sites, i.e. $\left[\mathrm{Pd}\left(\mathrm{NH}_{3}\right)_{4}\right]^{2+}$, inside a nickel pyrazolate porous framework. The resulting material has been employed as catalyst in hydroamination reactions, outperforming traditional inorganic supports. The high activity of the Pd@ NiBDP allows to work under mild reaction conditions, being possible to perform various reaction cycles without structural damage or dramatic activity decrease. The high selectivity towards the hydroamination of the alkyne function vs. the competing alkyne hydration reaction and the possibility of reuse it different times without major loss of activity or selectivity, makes this Ni-Pd pyrazolate an interesting system to catalyze the activation of other C-H bonds in organic synthesis. For this type of nucleophiles addition (amines, water) to triple bonds, it is possible to tune the selectivity of the Pd catalysts by its confinement inside porous supports with different composition, structure, crystal size and porosity (here only NiBDP MOF or HY Zeolite are studied). While the desired hydroamination reaction pathway is favoured in the presence of the NiBDP, the zeolite promotes the parallel hydration reaction.

\section{Acknowledgements}

The European Commission-Horizon 2020 under the Marie Sklodowska-Curie Individual Fellowship, grant agreement number 750391 (Project acronym SINMOF), is kindly acknowledged for financial support. 


\section{References}

1. Taylor AP, Robinson RP, Fobian YM, Blakemore DC, Jones LH, Fadeyi O. (2016) Modern advances in heterocyclic chemistry in drug discovery. Org. Biomol. Chem., 14: 6611-6637.

2. (a) Bechtoldt A., Tirler C, Raghuvanshi K, Warratz S, Kornhaaß C, Ackermann L (2016) Ruthenium Oxidase Catalysis for Site-Selective $\mathrm{C}-\mathrm{H}$ Alkenylations with Ambient $\mathrm{O} 2$ as the Sole Oxidant. Angew. Chem. Int. Ed., 55:264-267; (b) Ackermann L., (2005) General and Efficient Indole Syntheses Based on Catalytic Amination Reactions. Org. Lett. 7:439-442; (c) Ackermann L., Barfüßer S., Potukuchi HK. (2009) Copper-Catalyzed N-Arylation/Hydroamination Domino Synthesis of Indoles and its Application to the Preparation of a Chek1/KDR Kinase Inhibitor Pharmacophore. Adv. Synth. Catal., 351:1064-1072, (d) Ackermann L, Althammer A. (2007) Domino N-H/C-H bond activation: palladium-catalyzed synthesis of annulated heterocycles using dichloro(hetero)arenes. Angew. Chem. Int. Ed., 46:1627-1629.

3. Gates BC (2016) Concluding remarks: progress toward the design of solid catalysts. Faraday Discuss. 188:591-602.

4. (a) Cirujano FG (2017) MOFs vs. zeolites: carbonyl activation with M(IV) catalytic sites. Catal. Sci. Technol., 7: 5482-5494; (b) Santoro S, Kozhushkov SI, Ackermann L, Vaccaro L (2016) Heterogeneous catalytic approaches in $\mathrm{C}-\mathrm{H}$ activation reactions. Green Chem., 18:3471-3493; (c) Alonso F, Beletskaya IP, Yus M (2005) Non-conventional methodologies for transition-metal catalysed carbon-carbon coupling: a critical overview. Tetrahedron 61:11771-11835.

5. (a) Sachtler WMH, Cavalcanti FAP (1991) Coordination, atom reorganization, and catalysis of palladium in zeolite cages. Catal. Lett., 9:261-271; (b) Sachtler WMH (1993) Metal Clusters in Zeolites: An Intriguing Class of Catalysts. Acc. Chem. Res., 26:383-387; (c) Djakovitch L, Rollet P (2004) Adv. Synth. Catal. 346(13-15): 1782-1792; (d) Djakovitch L, Dufaud V, Zaidi R (2006) Heterogeneous Palladium Catalysts Applied to the Synthesis of 2- and 2,3-Functionalised Indoles. Adv. Synth. Catal. 348(6): 715-724; (e) Djakovitch L, Rouge P (2007) New homogeneously and heterogeneously $[\mathrm{Pd} / \mathrm{Cu}]$-catalysed $\mathrm{C} 3$-alkenylation of free $\mathrm{NH}$-indoles. J. Molec. Catal. A, 273: 230-239.

6. (a) Li H, Zhu Z, Zhang F, Xie S, Li H, Li P, Zhou X (2011) Palladium Nanoparticles Confined in the Cages of MIL-101: An Efficient Catalyst for the One-Pot Indole Synthesis in Water. ACS Catal., 1:1604-1612; (b) Manna K, Zhang T, Lin W (2014) Postsynthetic Metalation of BipyridylContaining Metal-Organic Frameworks for Highly Efficient Catalytic Organic Transformations. J. Am. Chem. Soc. 136:6566-6569; c) Huang YB., Shen M., Wang X., Huang P., Chen R., Lin ZJ, Cao R (2016) Water-medium $\mathrm{C}-\mathrm{H}$ activation over a hydrophobic perfluoroalkane-decorated metalorganic framework platform. J. Catal., 333:1-7.

7. (a) López-Maya E, Montoro C, Colombo V, Barea E, Navarro JAR (2014) Improved CO2 Capture from Flue Gas by Basic Sites, Charge Gradients, and Missing Linker Defects on Nickel Face Cubic Centered MOFs. Adv. Funct. Mater. 24:6130-6135; (b) Montoro C, Ocõn P, Zamora F, Navarro JAR (2016) Metal-Organic Frameworks Containing Missing-Linker Defects Leading to High Hydroxide-Ion Conductivity Chem. Eur. J. 2016, 22, 1646-1651; (c) Rodríguez-Albelo LM, LópezMaya E, Hamad S, Ruiz-Salvador R, Calero S, Navarro JAR, (2017) Selective sulfur dioxide adsorption on crystal defect sites on an isoreticular metal organic framework series. Nat. Commun. 8:14457; (d) Cirujano FG, López-Maya E, Rodríguez-Albelo M, Barea E, Navarro JAR, De Vos DE (2017) Selective One-Pot Two-Step C-C Bond Formation using Metal-Organic Frameworks with Mild Basicity as Heterogeneous Catalysts. ChemCatChem 9:4019-4023.

8. (a) Djakovitch L, Koehler K (2001) Heck Reaction Catalyzed by Pd-Modified Zeolites. J. Am. Chem. Soc. 123:5990-5999; (b) Noel S, Luo C, Pinel C, Djakovitch L (2007) Efficient Heterogeneously Palladium-Catalysed Heck Arylation of Acrolein Diethyl Acetal. Selective Synthesis of Cinnamaldehydes or 3-Arylpropionic Esters. Adv. Synth. Catal. 349(7):1128-1140.

9. (a) Augustyniak AW, Zawartka W, Navarro JAR, Trzeciak AM (2016) Palladium nanoparticles supported on a nickel pyrazolate metal organic framework as a catalyst for Suzuki and carbonylative Suzuki couplings. Dalton Trans. 45:13525-13531 (b) Fortea-Pérez FR, Mon M, Ferrando-Soria D, Boronat M, Leyva-Pérez A, Corma A, Herrera JM, Osadchii D, Gascon J, Armentano and E. Pardo (2017) The MOF-driven synthesis of supported palladium clusters with catalytic activity for carbene-mediated chemistry. Nat. Mater 16:760-766.

10. (a) Mueller TE, Hultzsch KC, Yus M, Foubelo F, Tada M (2008) Hydroamination: direct addition of amines to alkenes and alkynes. Chem. Rev. 108:3795-892. (b) Penzien J, Haeßner C, Jentys A, Köhler K, Müller TE, Lercher JA (2004) Heterogeneous catalysts for hydroamination reactions: structure-activity relationship. J. Catal. 221: 302-312; (c) Rubio-Marqués P, Rivero-Crespo MA, Leyva-Pérez A, Corma A (2015) Well-Defined Noble Metal Single Sites in Zeolites as an Alternative to Catalysis by Insoluble Metal Salts. J. Am. Chem. Soc. 137:11832-11837; (d) Marquez C, Rivera-Torrente M, Paalanen PP., Weckhuysen BM., Cirujano FG, De Vos D, De Baerdemaeker $\mathrm{T}$ (2017) Increasing the availability of active sites in $\mathrm{Zn}-\mathrm{Co}$ double metal cyanides by dispersion onto a SiO2 support. J. Catal. 354: 92-99.

11. Perry CH, Athans DP, Young EF (1967) Far Infrared spectra of palladium compounds. Spectrochimica Acta 173:1137-1147.

12. (a) Cabrero-Antonino JR, Leyva-Pérez A, Corma A (2015) Beyond Acid Strength in Zeolites: Soft Framework Counteranions for Stabilization of Carbocations on Zeolites and Its Implication in Organic Synthesis. Angew. Chem. Int. Ed. 54:5658-5661; (b) Cabrero-Antonino JR, Leyva-Pérez 
A, Corma A (2012) Chem. Eur. J. 18:11107-11114; (c) Liu W, Wang H, Li CJ (2016) Metal-Free Markovnikov-Type Alkyne Hydration under Mild Conditions. Org. Lett. 18: 2184-2187; (d) La Sorella G, Sperni L, Ballester P, Strukula G, Scarso A (2016) Hydration of aromatic alkynes catalyzed by a self-assembled hexameric organic capsule. Catal. Sci. Technol. 6:6031-6036.

13. Sing KSW, Everett DH, Haul RAW, Moscou L, Pierotti RA, Rouquerol J, Siemieniewska T (1985) Reporting physisorption data for gas/solid systems with Special Reference to the Determination of Surface Area and Porosity, Pure \& Appl. Chem. 57: 603-619.

14. Chen Q, Lv L, Yu M, Shi Y, Li Y, Pang G. Cao C (2013) Simple, efficient and reusable PdNHC catalysts for hydroamination. RSC Adv. 3: 18359-18366.

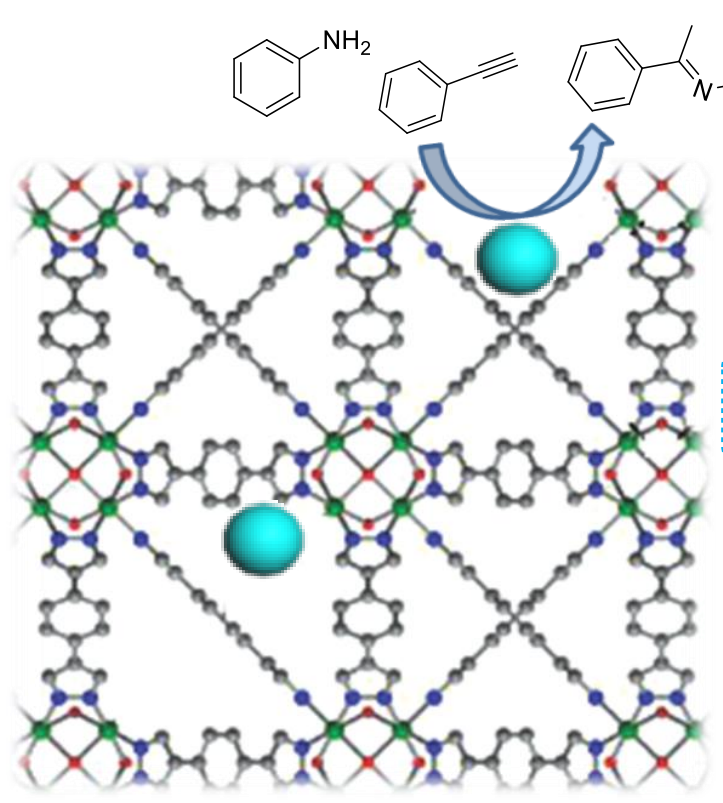

NiBDP-MOF
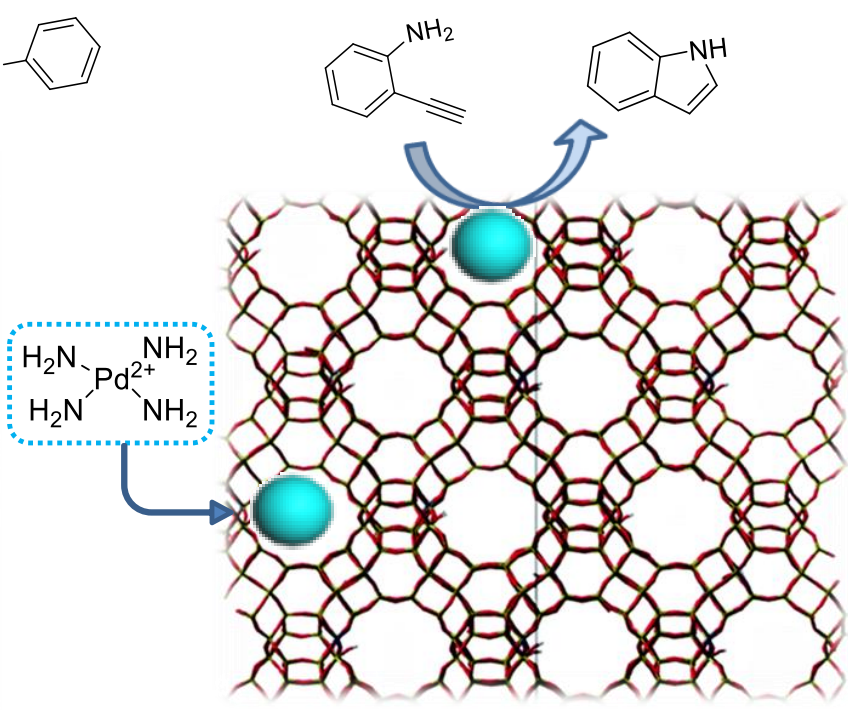

$1 \mathrm{~nm}$

HY-ZEOLITE 